\title{
Driving equipments made by ICPE SAERP for urban electric transport vehicles
}

\author{
V. Radulescu, I. Strainescu, L. C. Moroianu, V. Serbu, E. Tudor, \\ S. Gheorghe, C. Goia, Fl. Bozas, A. Dascalu, D. Braslasu, \\ M. Tanase, G. Mitroi, S. Badea, I. Sburlan, C. Ungurasu, V. Lupu \\ \& B. Radulescu \\ ICPE SAERP S.A, Bucharest, Romania
}

\begin{abstract}
The company ICPE SAERP S.A. is the main producer of the electric drives for urban traction and for railway vehicles in Romania. The products of our company are subject to the last 57 years of permanent evolution, based on the development of the semiconductor and of the microprocessor control techniques. The improvement of the passenger's comfort and the downsizing of the exploitation costs are a must for public transportation companies, relating to trolleybuses and trams. Both can be achieved by using modern electric drives (dc-choppers or 3-phase inverters), which can reduce the power consumption and can increase the control of the vehicle. The main products for electric traction are the drives for traction motors of the vehicles (as DC-choppers for DC series motors and 3-phase inverters for asynchronous or synchronous motors) and converters for auxiliary services of the vehicles (battery chargers and 3-phase inverters for auxiliary asynchronous motors). This paper represents a review of those applications and their benefits.
\end{abstract}

Keywords: electric drive, inverter, chopper, trolley, tram, converter, charger, research, development.

\section{Driving equipment for the traction motors}

\subsection{Choppers for direct current motors}

This chapter presents such a system that is mounted or can be mounted on the tramcar and on the trolleybus. ICPE SAERP S.A. has delivered 359 traction 
equipments with DC chopper based on GTO thyristors or IGBT transistors. All those equipments are trolleybuses from ASTRA BUS SRL Arad for the final beneficiaries RAT Bucharest, TRANSURB Galați and RATUC Cluj - Napoca.
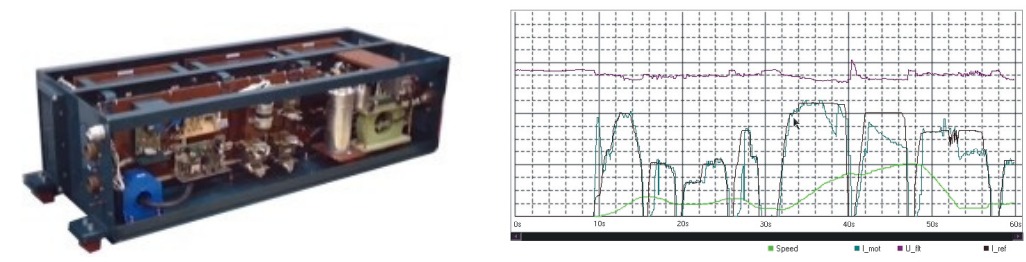

Figure 1: $\quad$ DC chopper unit with IGBT for trolleybuses and scope diagram.

Between 1995 and 1997, together with the specialists of RAT Bucharest, ICPE SAERP has equipped 22 trolleybuses with DC drives, by modifying some SAURER buses into trolleybuses. Most of them were in use until 2008. Between years 1997 and 2001, a number of 203 trolleybuses type ASTRAIK 415T were built at ASTRABUS Arad, RO. With the same partner we have produced 27 IRISBUS-AGORA trolleybuses for Cluj-Napoca, Romania, during the year 2002.
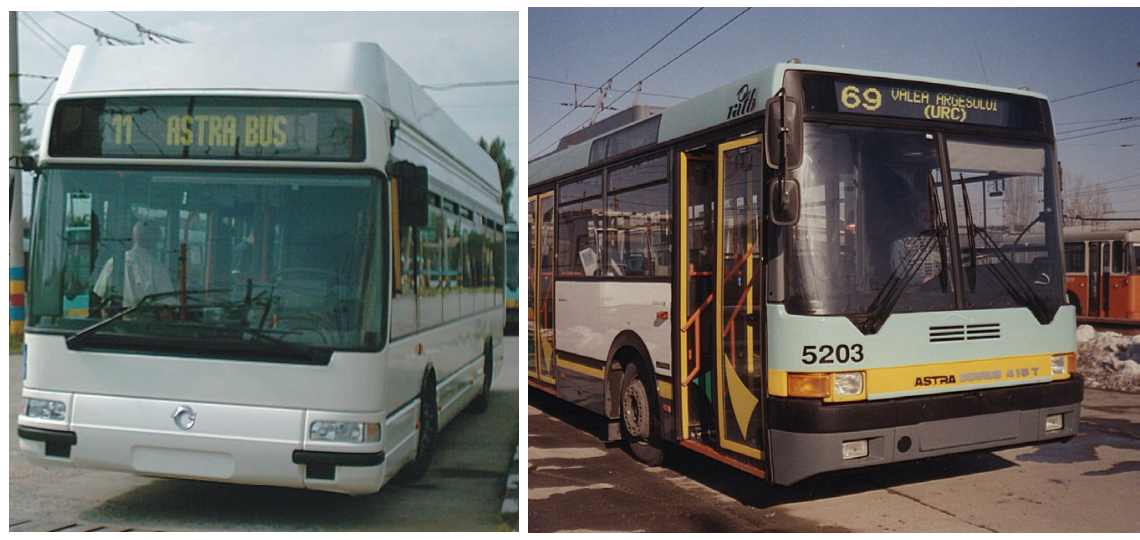

Figure 2: IRISBUS-ASTRABUS and ASTRAIK-415T trolley based on the DC chopper.

The production of years 2006-2008 consists in 100 trolleys type ASTRABUS-CITELIS for RAT Bucharest. Also, between 2002...2005, 4 COBRA trolleybuses have been built with TRAMKAR Sofia, Bulgaria.

The benefits of these vehicles are:

- $45 \%$ less energy consumption (related to an similar vehicle based on rheostat);

- The comfort increase due to the smooth control of the acceleration and the deceleration performed by the microprocessor unit; 
- The increase of the availability time by using high-reliability components and improved tests. The MTBF (medium time between failure) was increased up to 30000 hours;

- Cost downsizing regarding the maintenance.

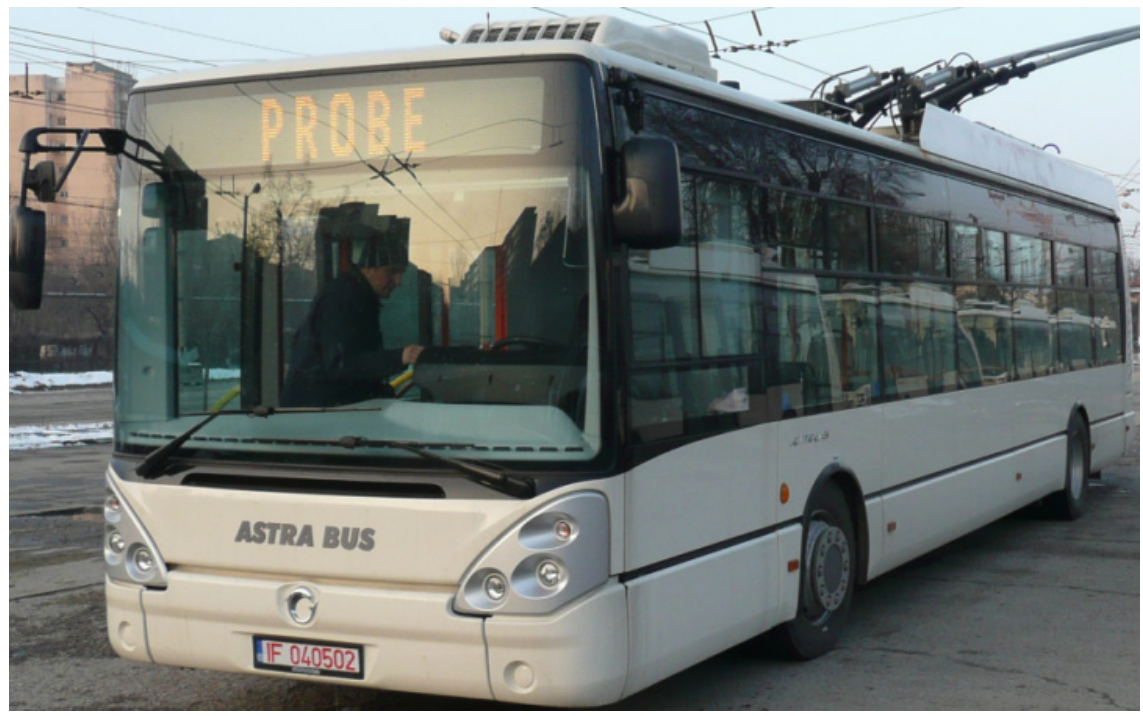

Figure 3: Astrabus-Citelis trolleybus for Bucharest.

An electronic unit called SATREC, based on microcontrollers, controls those chopper equipments. This unit consists in several electronic boards connected via a back plate. The master board (UCP) controls the communication on the back plate with several IO boards and with the UCS slave controller. The slave card UCS is a dedicated board, which performs the real time control of the power converter, in this case, the chopper.

Main functions of the SATREC-MMA control unit are:

- Digital control of the vehicle's traction system;

- Drives all electrical systems of the trolley bus, such as: switches, contactors, doors, stop brake, heaters;

- The system's diagnose and self diagnose;

- On-board display for real-time data as: energy consumption / energy regeneration, total trip $(\mathrm{km})$, service timer $(\mathrm{h})$, status.

The unit called SATREC MMA is the larger unit, which contain both the master and the slave controller. For distributed architecture, the master controller resides into the control unit SATREC-CGT, and the slave controller is located inside the frame of the chopper, into a different unit, named SATREC-CVT.

The distributed control system advantages are:

- Low wiring costs;

- Advanced regulator of traction torque

- Maximum speed digital regulator; 
- Acceleration and deceleration control;

- Slip control during traction and brake;

- Balanced charge of the traction motors;

- Driving system diagnosis;

- Complementary braking systems command, including station stop;

- Secondary driving desk (rear desk);

- Diagnosis on three levels, including the board display;

- Trip, energy consumption, recovered energy, service time counter;

- Event memory and trace memory, for the last $1000 \mathrm{~km}$;

- Interoperability, for use in train configuration as a light metro.

The Master-Slave architecture can be used in several configurations, as:

- $\mathrm{M}+\mathrm{S}+\mathrm{D}$, one master, one slave, one display, for trolley bus or small tram;

$-\mathrm{M}+2 * \mathrm{~S}+\mathrm{D}$, one master, two slaves, one display, for trams with 2 motors;

- $\mathrm{M}+2 * \mathrm{~S}+\mathrm{D}+\mathrm{M}+2 * \mathrm{~S}$, two masters, each with two slaves, one display, for train consists of two trams with 2 motors;

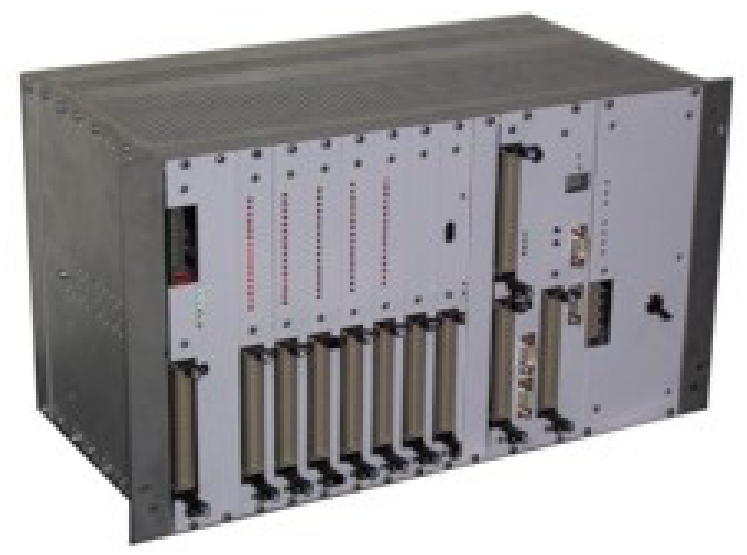

Figure 4: $\quad$ SATREC-MMA for the trolleybus.

The preferred serial connection between control units is RS485-type or CAN bus, with 1200 meters length capability. The transmission is half-duplex, asynchronous, and is controlled by the Active Master UCP, by TDMA approach (time division medium access).

This diagram was applied on the prototype of tram type $\mathrm{V} 3 \mathrm{~A}-93 \mathrm{CH}$, which was equipped with a single chopper which controls two motored bogies. The electronic control is SATREC-CGT as master control unit (with UCP), a slave unit SATREC-CVT located inside the chopper cubicle (on the roof) and a display type ADT07 on the driver's desk.

This type of driving equipment reliability was calculated to be of over 12000 hours of use between failures. This application is dedicated for the refurbishment of old trams with DC motors, and the costs can be subtracted after 2,5 years from the energy saved, lower maintenance costs and increased operability.

Another benefits of the numerical control units are the diagnose facilities. Diagnose of the distributed systems is divided, in such manner as a time- 
effective diagnose is located at the slave level, as the general event manager can be recorded at the Slave memory.

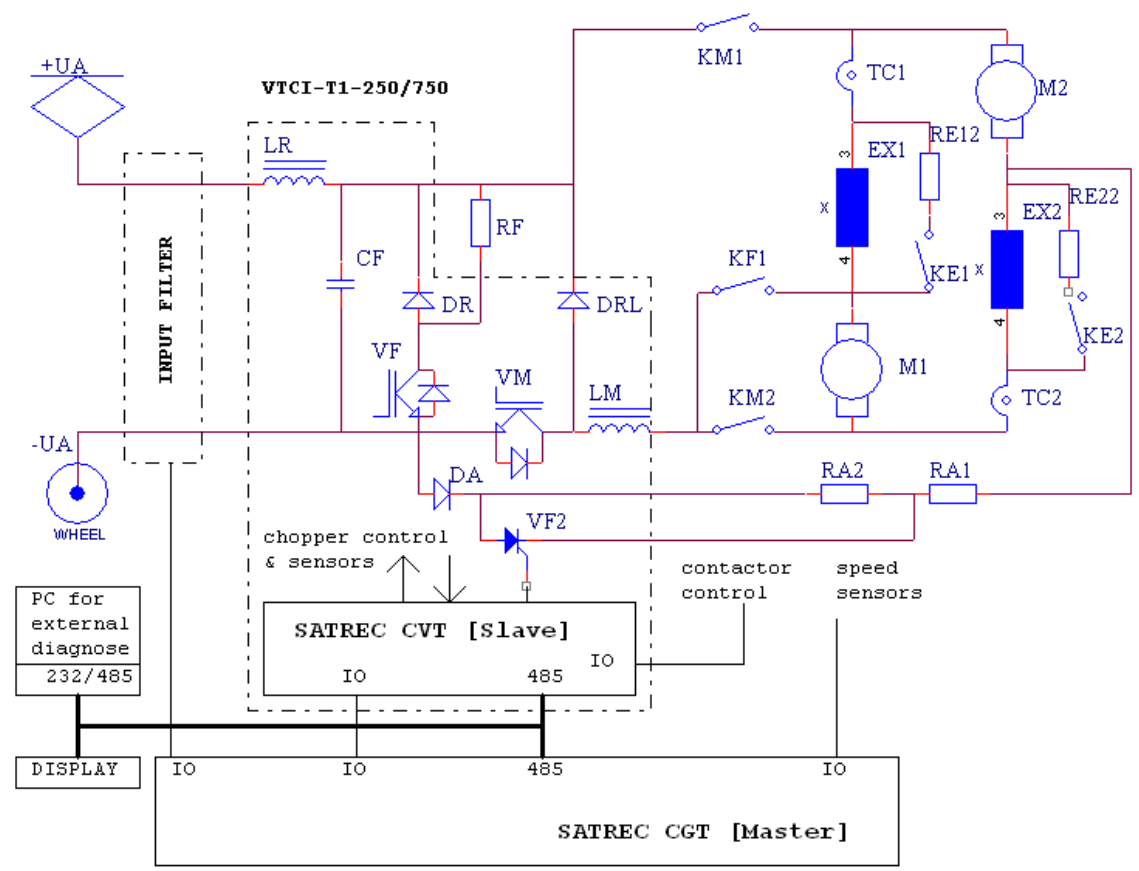

Figure 5: $\quad$ Electrical diagram for tram- single slave.

For diagnose we developed DCSoft software, especially designed for our drives. Each type of equipment uses a different version of software. The particularities and the multitude of functions of the diagnose software increases the operability of the software for the final user.

In the figure 6 is a caption of the DCSoft software, version 3.01, that version being designated to be used for the equipment presented in figure 5 .

\subsection{Three-phase inverters for asynchronous motors.}

Electrical traction with asynchronous motors driven by a three-phase inverter is the preferred solution for new vehicles.

The advantages of these systems are:

- Smaller number of traction contactors;

- The traction motor is more compact and reliable;

- Cabling system is reduced;

- The comfort of the passengers is improved;

- Braking torque is available at low speed.

The specific disadvantages of $\mathrm{AC}$ drives are:

- The traction inverter is complicated; 

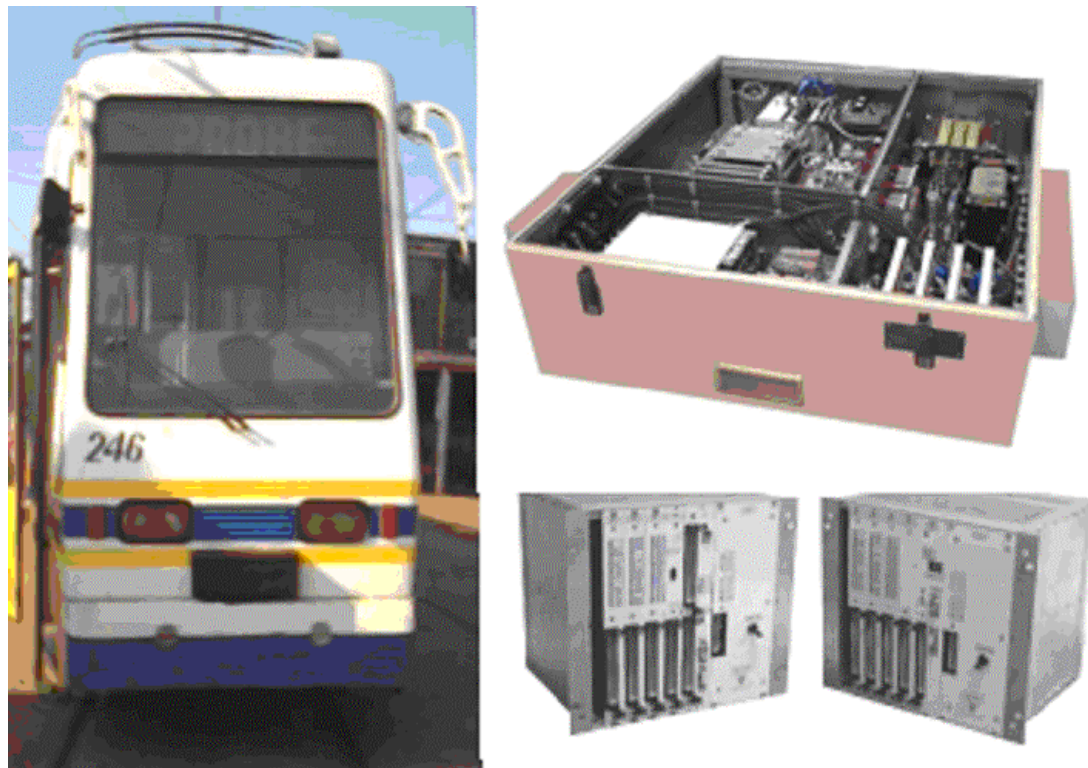

Figure 6: Tram, chopper cubicle and control units.

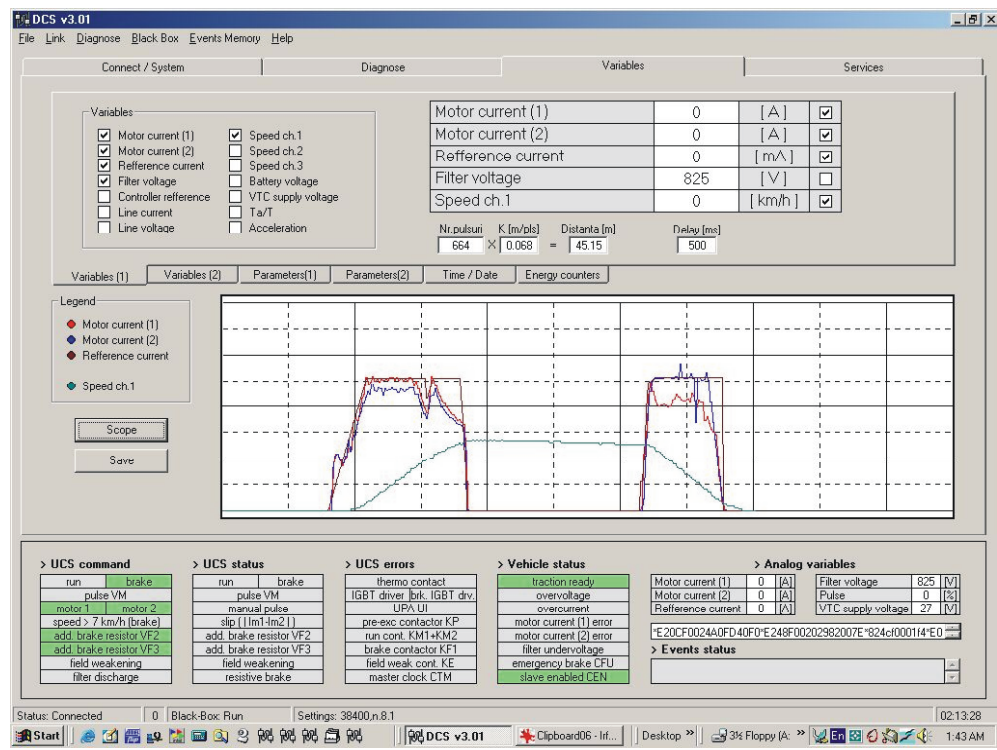

Figure 7: Tram test - DCSoft caption.

- The microprocessor command is also complicated;

- The motor needs a special construction from the point of view of isolation;

- The transducers must be very accurate.

The next picture represents the traction inverter for the trolleybus. 


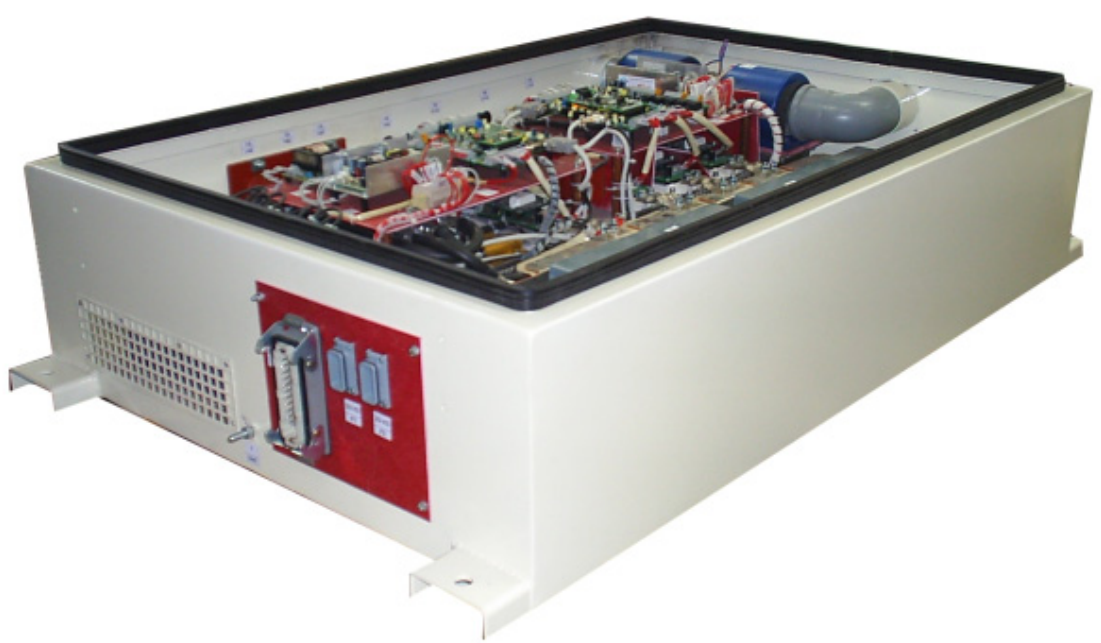

Figure 8: $\quad$ The traction inverter IVF.

The inverter IVF is provided with:

- On-board control unit with DSP;

- Built-in capacitor banks;

- Three-quadrant input rectifier;

- 24Vdc supply;

- permanent air cooling;

- On-line diagnose vian CAN-bus;

\section{Auxiliary static converters for urban traction vehicles}

The auxiliary converters are designated to provide:

- $24 \mathrm{Vdc}$ (or else) to supply low-voltage commands of the vehicle, including battery charger;

- $230 \mathrm{Vac}, 50 \mathrm{~Hz}$, for special appliances inside the vehicles;

- 3*400 Vac, $50 \mathrm{~Hz}$, for asynchronous motors used for steering, air compressor, power-cooling systems.

\subsection{Low-voltage supply and battery charger (24Vdc or $110 \mathrm{Vdc})$}

The static converters are used to convert the energy feed from the line (600 or $750 \mathrm{Vdc}$ ) to the low voltage circuits for trams. The output voltage is regulated and performs:

- Limit of the charging current of the battery and control the buffer voltage;

- Limit of the total supplied current;

- Voltage precision - 2\%;

For this product the determined MTBF number is over 16.000 functioning hours. 


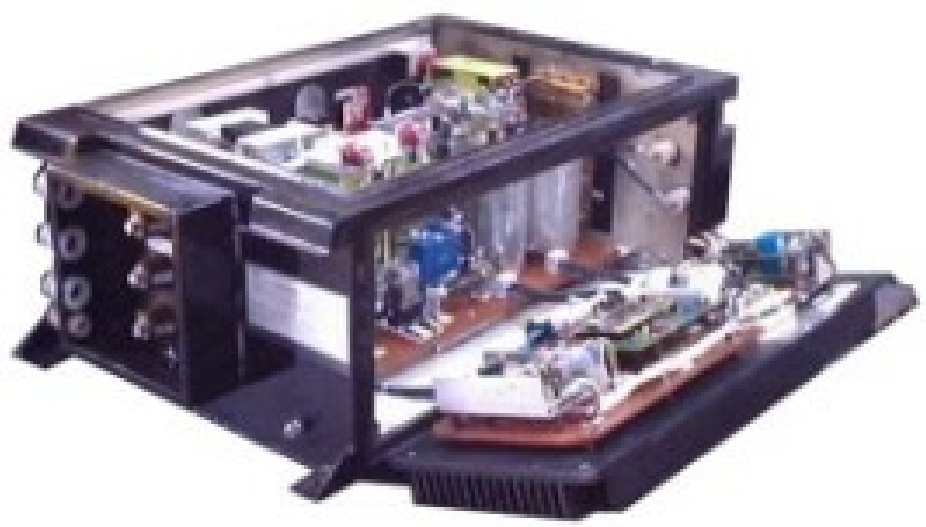

Figure 9: $\quad$ Auxiliary tram converter SIF 28/750.

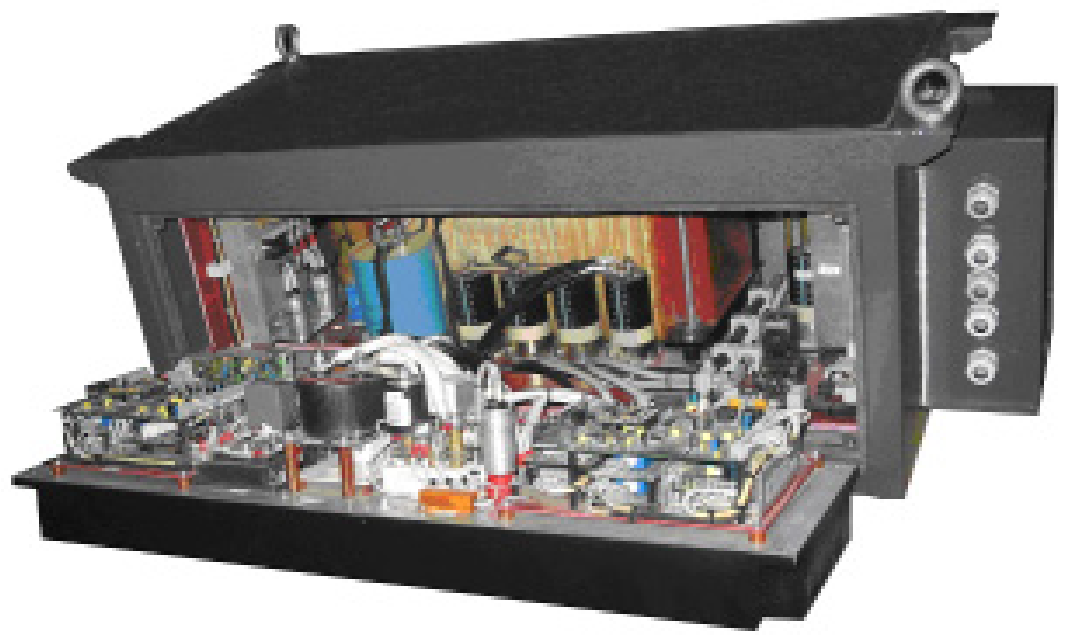

Figure 10: Static converter with dual output.

\subsection{Dual output static converter}

The static converters for the auxiliary services on trolleybuses have two output circuits; both of them are double-isolated galvanic towards the supply voltage:

- 28Vcc - low voltage and battery charging output;

- 3x400Vca - Asynchronous motors output for auxiliary services (servo direction, compressor, ventilation).

The three-phase output is usually described by:

- Fixed frequency and fixed voltage in stationary regime;

- Constant torque during start up (U/f=constant);

- DC voltage compensation; 
- The output current and voltage are sinusoidal, with an $8 \%$ THD;

- Overload, short-circuit and overheat protection.

The MTBF determined number is over 12.000 functioning hours.

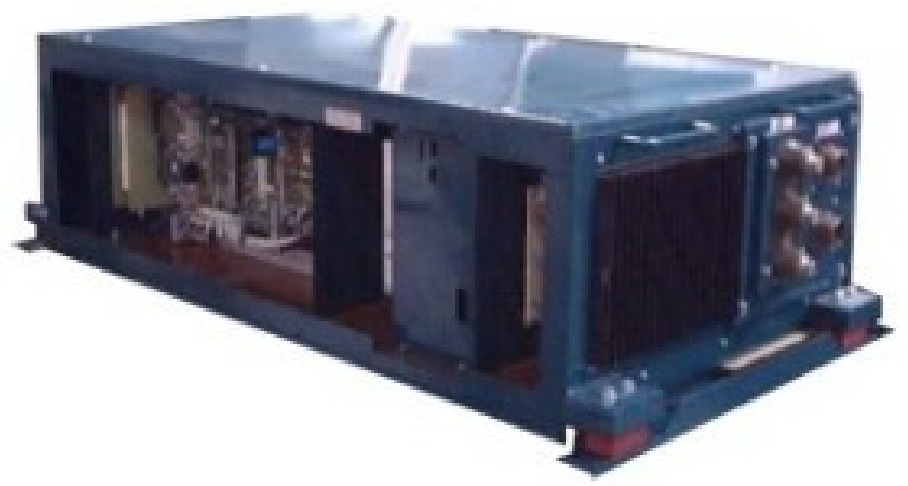

Figure 11: Static converter for auxiliary services on trolley buses CS 11T.

\section{Conclusions and observations}

The equipment is developed for trams and trolleybuses, and it must fulfil both the IEC requirements for trams and the E/ECE/TRANS prescriptions regarding trolley buses. A high durability, the possibility of isolating the damaged vehicle and reduced maintenance and diagnosis operations are needed.

Having these to follow, the research, design and production activity of ICPE SAERP S.A. has closely monitored the evolution of the semiconductor devices and materials to increase the technical level of the products.

The driving command is made entirely within the company, starting with the design of the printed circuits and equipping the electronic boards, development of the control, communication and diagnosis software implemented at the level of command electronics all the way to the diagnosis software on the PC regarding the human-machine interface.

ICPE SAERP S.A. has presented the research and production activity by participating at fairs, expositions, communications at intern and international scientific reunions. Also, the news is presented on the internet site www.saerp.ro that allows the "on-line" update of the diagnosis software. 